\title{
CD90 is upregulated in gastric cancer tissues and inhibits gastric cancer cell apoptosis by modulating the expression level of SPARC protein
}

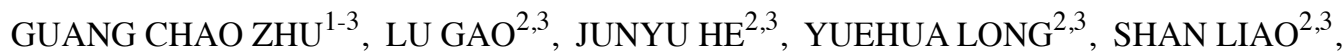 \\ HAIYUN WANG ${ }^{4}$, XUJUAN $I^{4}$, WEI YI ${ }^{2,3}$, ZHEN PEI ${ }^{2,3}$, MINGHUA WU ${ }^{1-3}$, JUANJUAN XIANG ${ }^{1-3}$,

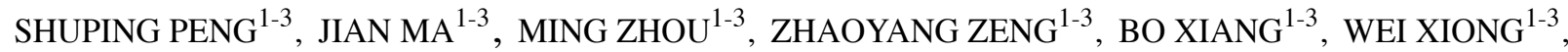

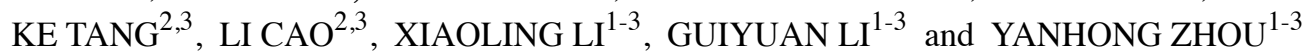

\begin{abstract}
${ }^{1}$ Hunan Provincial Tumor Hospital and the Affiliated Tumor Hospital of Xiangya School of Medicine, Central South University, Changsha, Hunan $410006 ;{ }^{2}$ Cancer Research Institute, Central South University;

${ }^{3}$ Key Laboratory of Carcinogenesis of the Ministry of Health and Key Laboratory of Carcinogenesis and Cancer Invasion of the Ministry of Education, Cancer Research Institute, Central South University, Changsha, Hunan 410078; ${ }^{4}$ School of Life Science and Technology, Tongji University, Shanghai 200092, P.R. China
\end{abstract}

Received June 30, 2015; Accepted July 24, 2015

DOI: $10.3892 /$ or. 2015.4243

\begin{abstract}
Cluster of differentiation 90 (CD90) (Thy-1) plays important roles in the oncogenesis in various types of malignancies. In the present study, we investigated the expression of CD90 in gastric cancer (GC) tissues by q-PCR, immunohistochemistry (IHC), and western blot technologies. The results showed that CD90 was overexpressed in gastric cancer tissues compared with the level in the adjacent non-cancerous tissues. To explore the possible mechanism of CD90 in GC, we elucidated the effect of CD90 on the apoptosis of AGS gastric cancer cells, and found that a considerable decrease in apoptotic cells was observed for AGS cells with CD90 overexpression. Meanwhile, the rate of apoptotic cells was increased in the AGS cells with CD90 interference (siCD90) compared with that in the AGS cells. Cell apoptosis is closely related to a reduction in mitochondrial membrane potential $(\Delta \Psi \mathrm{m})$ and an increase in intracellular reactive oxygen species (ROS)
\end{abstract}

Correspondence to: Dr Yanhong Zhou or Professor Guiyuan Li, Cancer Research Institute, Central South University, 10 Xiangya Road, Changsha, Hunan 410078, P.R. China

E-mail: zhouyanhong@csu.edu.cn

E-mail:1gy@csu.edu.cn

Abbreviations: GC, gastric cancer; Thy-1 (also known as CD90), Thy-1 cell surface antigen; SPARC, secreted protein, acidic, cysteine-rich (osteonectin); ROS, reactive oxygen species; FBS, fetal bovine serum; DCFH-DA, 2',7'-dichlorofluorescein diacetate; PI, propidium iodide; IHC, immunohistochemistry; GADPH, glyceraldehyde-3-phosphate dehydrogenase

Key words: CD90, gastric cancer, apoptosis, SPARC and calcium ion $\left(\mathrm{Ca}^{2+}\right)$ concentrations. Our results showed that overexpression of CD90 in the AGS gastric cancer cells led to an increase in $\Delta \Psi \mathrm{m}$ and a decrease in intracellular ROS and $\mathrm{Ca}^{2+}$ concentrations. At the same time, siCD 90 reduced $\Delta \Psi \mathrm{m}$ and the increase in intracellular ROS and $\mathrm{Ca}^{2+}$ concentrations. Furthermore, we identified and confirmed that CD90 functions by modulating the expression level of secreted protein, acidic, cysteine-rich (osteonectin) (SPARC) in vitro through LC-MS/MS analyses and western blot technology. In summary, our results suggest that CD90 is upregulated in gastric cancer and inhibits gastric cancer cell apoptosis by modulating the expression level of SPARC protein.

\section{Introduction}

Cluster of differentiation 90 (CD90) is a 25- to 37- kDa heavily $\mathrm{N}$-glycosylated, glycophosphatidylinositol anchored conserved cell surface protein with a single V-like immunoglobulin domain, originally discovered as a thymocyte antigen (1-3). In humans, CD90 is expressed by endothelial cells (ECs), smooth muscle cells, a subset of $\mathrm{CD} 34^{+}$bone marrow cells, cardiac fibroblasts and fetal liver-derived hemopoietic cells (4-6). As a tumor marker, CD90 has been shown to have significantly high expression in esophageal cancer and other digestive cancers $(7,8)$. CD90 expression was found to be valuable in the differential diagnosis between epithelioid mesothelioma and lung adenocarcinoma $(2,9)$. CD90 contributes to the metastasis of melanoma cells by mechanisms likely involving a CD90-mediated adhesion of melanoma cells to ECs $(4,10,11)$. CD90 could serve as a promising marker for pancreatic adenocarcinoma where desmoplastic stroma plays an important role in tumor growth and angiogenesis (12). CD90 was found to be expressed in $95 \%$ of clinical gastric tumor samples by immunohistochemical staining. $\mathrm{CD} 90^{+}$cells possess a higher ability to initiate tumors in vivo and self-renewal properties. 
These cells could be undermined by trastuzumab treatment in vivo $(13,14)$. Although some evidence has been reported, the mechanism of CD90 in gastric cancer is not fully understood.

Cell apoptosis is a crucial mechanism for all multicellular organisms to control cell proliferation and maintain tissue homeostasis (15-17). Identification of the mechanisms of apoptosis and the effector molecules responsible for apoptosis has provided a new opportunity to explore and develop novel agents which can increase the sensitivity of cancer cells to undergo apoptosis or reset their apoptotic threshold $(15,18,19)$. Cell apoptosis is closely related with a reduction in mitochondrial membrane potential $(\Delta \Psi \mathrm{m})$ and an increase in intracellular reactive oxygen species (ROS) and calcium ion $\left(\mathrm{Ca}^{2+}\right)$ concentrations. Mitochondria are important cellular structures that supply cellular energy by generating adenosine triphosphate (ATP) (20). Furthermore, mitochondria act as internal calcium stores through the uptake of intracellular-free $\mathrm{Ca}^{2+}$, and this is critical for calcium buffering (20). Mitochondrial calcium buffering is important during continuous calcium-induced calcium release in submandibular acinar cells, directly modulates agonist-induced calcium signals, and can functionally interact with calcium stores to regulate cytosolic calcium signals. When mitochondrial membranes are damaged, mitochondria lose their calcium buffering capability and mitochondria-related proteins, such as cytochrome $c$ and Smac/DIABLO, are released from the mitochondria into the cytosol, resulting in cellular apoptosis (21-25).

In this study, we examined the expression levels of CD90 in gastric cancer tissues. At the same time, we studied the influence of CD90 on cell apoptosis and explored the possible mechanism in a gastric cancer cell line.

\section{Materials and methods}

Cell culture. One identified general human gastric cancer cell line AGS, was cultured in Ham's/F-12 (HyClone, Logan, UT, USA) supplemented with $10 \%$ fetal bovine serum (FBS) (Gibco Life Technologies ${ }^{\mathrm{TM}}$, Grand Island, NY, USA), $100 \mathrm{U} / \mathrm{ml}$ penicillin and $100 \mu \mathrm{g} / \mathrm{ml}$ streptomycin (GE Healthcare Life Sciences, Logan, UT, USA) at $37^{\circ} \mathrm{C}$ in the presence of $5 \% \mathrm{CO}_{2}$.

Patient samples. Twelve participants were recruited at Xiangya Hospital, Central South University (Changsha, Hunan, China). Consent forms were obtained from individual patients, and experimental protocols were approved by the Institutional Review Board of Xiangya Hospital. All subjects enrolled in the study were Chinese. All clinical and biological data were available for the samples (Table I). Gastric cancer tissues and corresponding non-tumor normal tissues were collected, and each biopsy sample was divided into two sections; one was submitted for routine histological diagnosis and the remaining section was used for q-PCR, immunohistochemistry and western blot experiments.

Total RNA extraction and quantitative real-time PCR (qRT-PCR) analysis. Total RNA was extracted from the gastric cancer tissues and corresponding non-tumor normal tissues using TRIzol reagent (CWBio, Beijing, China) and cDNA synthesis was carried out using the RevertAid First Strand cDNA synthesis kit (CWBio) according to the manu- facturer's recommendations. qRT-PCR was carried out with GoTaq qPCR Master Mix (Promega, Fitchburg, WI, USA). For detection of CD90 mRNA expression levels, GAPDH was amplified in parallel as an internal control. The sequences of the primers used for qPCR were as follows: CD90 forward, 5'-gcatgggctaaggatttgaa-3' and reverse, 5'-tcccaaatttagcctgttgg-3'; GAPDH forward, 5'-cgaccactttgtcaagctca-3' and reverse, 5'-actgagtgtggcagggactc-3'. The expression of mRNA was assessed by evaluated threshold cycle (CT) values. The CT values were normalized to the expression levels of GAPDH and the relative amount of mRNA specific to each of the target genes was calculated using the $2^{-\Delta \Delta C t}$ method (26-31). qPCR was carried out using the Bio-Rad CFK96 ${ }^{\mathrm{TM}}$ Real-Time System (Bio-Rad, Hercules, CA, USA). The data were analyzed by Bio-Rad CFK Manager software (Bio-Rad). Expression of mRNA was assessed by evaluated CT values and GAPDH was used as an internal control.

Immunohistochemistry (IHC) and evaluation of staining. Immunohistochemistry was conducted using the peroxidase anti-peroxidase technique following a microwave antigen retrieval procedure. The antibody for $C D 90$ was purchased from Boster Biotechnology Co., Ltd. (Wuhan, China). The antibody against $C D 90$ (1:100) was overlaid on gastric cancer and corresponding non-tumor normal tissue sections and incubated overnight at $4^{\circ} \mathrm{C}$. Secondary antibody incubation (Santa Cruz Biotechnology, Inc., Santa Cruz, CA, USA) was performed at room temperature for $30 \mathrm{~min}$. Color reaction was developed using 3,3'-diaminobenzidine tetrachloride (DAB) chromogen solution. All slides were counterstained with hematoxylin. Positive control slides were included in every experiment in addition to the internal positive controls. The specificity of the antibody was determined with a matched $\mathrm{IgG}$ isotype antibody as a negative control.

Sections were evaluated by two investigators in a blinded manner in an effort to provide a consensus on staining patterns by light microscopy (Olympus, Tokyo, Japan). CD90 staining was assessed according to the methods described by Hara and Okayasu (32) with minor modifications. Each case was rated according to a score that was the sum of a scale of intensity of staining and the area of staining. At least 10 high-power fields were chosen randomly, and >1,000 cells were counted for each section. The intensity of staining was graded on the following scale: 0 , no staining; $1+$, mild staining; $2+$, moderate staining; $3+$, intense staining. The area of staining was evaluated as follows: 0 , no staining of cells in any microscopic fields; $1+,<30 \%$ of the tissue was stained positive; $2+$, between 30 and $60 \%$ was stained positive; $3+,>60 \%$ stained positive. The minimum score when summed (extension + intensity) was 0 and the maximum, 6 . A combined staining score (extension + intensity) of $\leq 2$ was considered to be negative staining (low staining); a score between 3 and 4 was considered to be moderate staining; whereas a score between 5 and 6 was considered to be strong staining. An optimal cut-off level was identified as follows: a staining index score of 0-2 was used to define tumors with negative expression and 3-7 indicated positive expression of these two proteins. Agreement between the two evaluators was $95 \%$, and all scoring discrepancies were resolved through discussion between the two evaluators. 
Table I. Characteristics of the gastric cancer patients.

\begin{tabular}{ccll}
\hline Patient no. & Age (years) & Gender & Histological diagnosis \\
\hline 1 & 54 & Male & Gastric poorly differentiated adenocarcinoma \\
2 & 59 & Male & Gastric poorly differentiated adenocarcinoma \\
3 & 63 & Female & Gastric intermediately differentiated adenocarcinoma \\
4 & 53 & Female & Gastric poorly differentiated adenocarcinoma \\
5 & 71 & Male & Gastric intermediately differentiated adenocarcinoma \\
6 & 67 & Male & Gastric poorly differentiated adenocarcinoma \\
7 & 55 & Male & Gastric intermediately differentiated adenocarcinoma \\
8 & 58 & Female & Gastric poorly differentiated adenocarcinoma \\
9 & 70 & Male & Gastric poorly differentiated adenocarcinoma \\
10 & 61 & Female & Gastric poorly differentiated adenocarcinoma \\
11 & 51 & Male & Gastric intermediately differentiated adenocarcinoma \\
12 & 62 & Male & Gastric poorly differentiated adenocarcinoma \\
\hline
\end{tabular}

Construction of the pEGFP-N1-CD90 vector and cell transfection. The coding region of the CD90 gene was generated by PCR with the primer pair 5'-atactcgaatgaacctggccatcagcat-3' and 5'-gcggaattctcacagggacatgaaatccg-3'. PCR was performed under the following conditions: one cycle for $5 \mathrm{~min}$ at $94^{\circ} \mathrm{C}$; 30 cycles for $45 \mathrm{sec}$ at $94^{\circ} \mathrm{C}, 45 \mathrm{sec}$ at $55^{\circ} \mathrm{C}$, and $90 \mathrm{sec}$ at $72^{\circ} \mathrm{C}$ and ending with $10 \mathrm{~min}$ at $72^{\circ} \mathrm{C}$. The fragments were cloned into the TA vector (Promega, Fitchburg, WI, USA) and used to transform E. coli JM109 (Takara, Dalian, China). Following selection and propagation, the pure plasmid DNA was prepared by standard methods. The DNA fragments were removed from the TA vector by restriction enzyme digestion with $X h \mathrm{oI}$ and EcoR1 (Promega) to subclone into the pEGFP-N1 vector. The fusion sequences were verified by DNA sequencing using ABI 3730. To establish a stable CD90-expressing cell line, the plasmid pEGFP-N1/CD90 or control empty vector pEGFP-N1 was transfected into AGS cells, using Lipofectin (Invitrogen Life Technologies, Carlsbad, CA, USA) according to the manufacturer's instruction, followed by G418 selection. The stable transfectants, AGS/CD90 and AGS/vector, were isolated, and transcription of the CD90 protein was determined by $\mathrm{qPCR}$ and western blot experiments.

Synthesis and transfection of siRNAs. siRNAs were designed and synthesized by RiboBio (Guangzhou, China). The siRNAs targeting the CD90 gene were designed and synthesized. The most effective siRNA (siCD90) identified by qPCR was applied for subsequent experiments. The sequence of siCD90 was: sense, 5'-UCCAGGCCACGGAUUUCAU dTdT-3' and antisense, 3'-dTdTAGGUCCGGUGCCUAA AGUA-5'. Twenty-four hours prior to transfection, the cells were plated onto a 6-well plate (Greiner, Stuttgart, Germany) at 40-60\% confluency. Transfection was performed with Lipofectamine 2000 (Invitrogen Life Technologies) according to the manufacturer's protocol. The medium was replaced 4-6 $\mathrm{h}$ after transfection with new culture medium, except in the case of AGS cells, for which the medium was not replaced after transfection. After an additional $48 \mathrm{~h}$ of culture, the cells were harvested for the following apoptosis, $\mathrm{Ca}^{2+}$ and ROS experiments.
Effect of CD90 on gastric cancer cell apoptosis. Cell apoptosis was analyzed by flow cytometric analysis using a Moflo ${ }^{\mathrm{TM}}$ XDP High-Performance Cell Sorter (Beckman Coulter, Brea, CA, USA), propidium iodide (PI) and Hoechst 33342 doublestaining (Nanjing KeyGEN Biotech Co., Ltd., Nanjing, China). Briefly, AGS cells (AGS, AGS/vector, AGS/CD90) were seeded at a density of $1 \times 10^{6}$ cells per well in 6-well culture plates. The cells were collected in an Eppendorf tube $(24 \mathrm{~h})$ and washed twice with PBS by centrifugation. The supernatants were discarded. To detect apoptosis, $500 \mu \mathrm{l}$ of PBS, $5 \mu \mathrm{l}$ of Hoechst 33342 and $5 \mu \mathrm{l}$ PI were added to each tube, and the contents of the tube were mixed in the dark at room temperature for $15 \mathrm{~min}$, followed by FCM testing (Beckman Coulter). Data were acquired and analyzed with Summit v5.2 software (Beckman Coulter).

Detection of mitochondrial membrane potential by JC-1. The impact of CD90 was measured by flow cytometry using the sensitive and relatively mitochondrion-specific lipophilic cationic probe fluorochrome JC-1. JC-1 accumulates to form J-aggregates and emits red fluorescence in the mitochondria with higher membrane potential, yet dissociates into monomers and emits green fluorescence in those that lose cross-membrane electrochemical gradient. The cells were suspended in $1 \mathrm{ml}$ warm staining buffer at $\sim 1 \times 10^{6}$ cells $/ \mathrm{ml}$ and incubated at $37^{\circ} \mathrm{C}$ for $5 \mathrm{~min}$. Then $1 \mu \mathrm{l}$ of $2 \mathrm{mM} \mathrm{JC}-1$ ( $2 \mu \mathrm{M}$ final concentration was added) and the cells were incubated at $37^{\circ} \mathrm{C}$ in $5 \% \mathrm{CO}_{2}$ for $15-30 \mathrm{~min}$. The cells were pelleted by centrifugation, resuspended by gently flicking the tubes, and $500 \mu 1$ PBS was added to each tube. Cells were analyzed with Moflo ${ }^{\mathrm{TM}}$ XDP High-Performance Cell Sorter (Beckman Coulter). Data were acquired and analyzed with Summit v5.2 software.

Intracellular ROS measurement. The production of intracellular reactive oxygen species (ROS) was measured by performing flow cytometry using the oxidation-sensitive probe, 2',7'-dichlorofluorescein diacetate (DCFH-DA) (Applygen, Beijing, China). Briefly, $10 \mathrm{mM}$ DCFH-DA stock solution (in methanol) was diluted 4,000-fold in cell culture medium without serum or other additive to yield a $2.5 \mathrm{mM}$ working 
solution. After the exposure of HUVECs to silica nanoparticles for $3 \mathrm{~h}$ and $24 \mathrm{~h}$, respectively, the cells in 6-well plates were washed twice with PBS and incubated in $2 \mathrm{ml}$ working solution of DCFH-DA at $37^{\circ} \mathrm{C}$ in the dark for $30 \mathrm{~min}$. Then the cells were washed twice with cold PBS and resuspended in PBS for analysis of intracellular ROS by FACS (Beckman Coulter).

Intracellular $\mathrm{Ca}^{2+}$ concentration assay. Intracellular $\mathrm{Ca}^{2+}$ concentration was measured by means of the fluorescent $\mathrm{Ca}^{2+}$ chelator Fura-2 AM, which permeates into cells where it is cut into Fura-2. Fura-2 combines with intracellular $\mathrm{Ca}^{2+}$ to form a fluorescent compound, whose fluorescent intensity was determined at an excitation wave length of $340 \mathrm{~nm}$ and an emission wave length of $510 \mathrm{~nm}$ in FACS (Beckman Coulter). After treatment, the cells were harvested and rinsed with PBS. The harvested cells were suspended in PBS and incubated with $5 \mu \mathrm{M}$ Fura- $2 \mathrm{AM}$ for $60 \mathrm{~min}$ at $37^{\circ} \mathrm{C}$. During the session of incubation with Fura-2 AM, cell cultures were mildly shaken at intervals of $10 \mathrm{~min}$ aimed to facilitate the combination of Fura-2 and $\mathrm{Ca}^{2+}$ to form the fluorescent compound. Then, cells were washed twice and resuspended in PBS for FACS measurement (Beckman Coulter). Data were acquired and analyzed with Summit v5.2 software (Beckman Coulter).

Identification of co-expressed genes by bioinformatic analysis. The RNA-Seq data of stomach adenocarcinoma, including 274 tumor samples and 33 normal samples, were retrieved from the Cancer Genome Atlas (TCGA). We obtained these data from Broad GDAC FIREHOSE (https://confluence. broadinstitute.org/display/GDAC/Home) on 2014-05-18. In these data, gene expression index was quantified and normalized using reads per kilobase per million reads (RPKM).

The Spearman's correlation $\left(\mathrm{r}_{\mathrm{s}}\right)$ (33) was introduced to measure the co-expression between gene CD90 and another gene $\left(g_{\mathrm{y}}\right)$ across the stomach samples. For a given sample size $\mathrm{n}$, we defined $\mathrm{X}_{\mathrm{i}}$ as the expression index of gene CD90 and $Y_{i}$ as the expression index of gene $g_{y}(i=1.2 n)$. Raw expression indices $X_{i}$ and $Y_{i}$ were converted to ranks $X_{i}$ and $y_{i}$, and $r_{s}$ was defined as follows: Where is the difference between ranks (?)(clarify) To explore the biological relationships of genes that were co-expressed with CD90, a functional enrichment analysis was introduced using DAVID (34). The goal of the enrichment analysis was to determine the biological functions that might be associated with CD90, as well as to narrow down various co-expressed genes that were mapped onto the specific functions concerned. We employed the Gene Ontology, KEGG and other functional categories as the background knowledge base to acquire the functional concepts for those co-expressed genes.

Identification of differential proteins in CD90-overexpressed AGS cells by LC-MS/MS analysis. The cell lysate was obtained using a protein extraction buffer consisting of $50 \mathrm{mM}$ Tris (pH 7.4), $150 \mathrm{mM} \mathrm{NaCl}, 1 \%$ Triton $\mathrm{X}-100,1 \%$ sodium deoxycholate, $0.1 \%$ SDS and sodium orthovanadate, sodium fluoride, EDTA, leupeptin supplemented with $1 \mathrm{X}$ halt protease inhibitor cocktail (CWBio) and 1X halt phosphatase inhibitor cocktail (BestBio, Shanghai, China). The protein concentration was estimated by the bicinchoninic acid (BCA) method. The gel was run at $80 \mathrm{~V}$ for $40 \mathrm{~min}$, then $120 \mathrm{~V}$ for $90 \mathrm{~min}$. Then, $50 \mu \mathrm{g}$ of each preparation was loaded onto a $10 \%$ SDS-PAGE gel. SDS-PAGE was run at $80 \mathrm{~V}$ for $40 \mathrm{~min}$, then $120 \mathrm{~V}$ for $90 \mathrm{~min}$. Protein bands were visualized using Coomassie brilliant blue G-250 (Sigma-Aldrich, Carlsbad, CA, USA) and excised. The protein spots were destained using $15 \mathrm{mM} \mathrm{K}_{4} \mathrm{Fe}(\mathrm{CN})_{6}$ and $50 \mathrm{mM}$ sodium thiosulfate and $1.25 \mu \mathrm{g}$ trypsin (1:20 enzyme/substrate ratio) was added to each band and in-gel digestion was performed at $37^{\circ} \mathrm{C}$ overnight $(\sim 16 \mathrm{~h})$. The generated peptides were extracted by sonication ( $15 \mathrm{~min}$, ice cooling) of the gel pieces in $\sim 20 \mu 1$ of $50 \%$ acetonitrile in $0.1 \% \mathrm{FA}$, twice. After extraction from the gel pieces, the peptides were dried by vacuum centrifugation to ensure a complete removal of acetonitrile and then reconstituted in $20 \mu 10.1 \%$ FA (35-37).

LC-MS/MS analyses were performed on an Ultimate ${ }^{\mathrm{TM}}$ 3000 RSLC Nano system online coupled to an LTQ Orbitrap Velos Pro mass spectrometer (both from Thermo Scientific, Bremen, Germany). Peptides were diluted with $0.1 \%$ FA; for each analysis $30 \mu 1$ of sample was injected. After injection, peptides were pre-concentrated with $0.1 \% \mathrm{FA}, 3 \% \mathrm{ACN}$ on a trap column ( $\mu$-Precolumn C18 PepMap 100,300 $\mu \mathrm{m} \times 5 \mathrm{~mm}$, $5 \mu \mathrm{m}, 100 \AA \AA$; Thermo Scientific) at a flow rate of $300 \mathrm{nl} / \mathrm{min}$ for $5 \mathrm{~min}$. Subsequently, the analyte was transferred to the analytical column (Acclaim ${ }^{\circledast}$ PepMap RSLC, $75 \mu \mathrm{m}$ x $15 \mathrm{~cm}$, nano Viper, C18, $2 \mu \mathrm{m}, 100 \AA$ A. Thermo Scientific) and separated using a 120 min gradient from 5 to $40 \%$ solvent $B$ at a flow rate of $300 \mathrm{nl} / \mathrm{min}$ (solvent A: $0.1 \%$ formic acid, solvent B: $0.08 \%$ FA $80 \%$ acetonitrile). The mass spectrometer was operated in a data-dependent mode. The general mass spectrometric parameters were as follows: spray voltage, $2.0 \mathrm{kV}$; capillary temperature, $275^{\circ} \mathrm{C}$. For data-dependent MS/MS analyses, the software XCalibur (Thermo Fisher Scientific, Waltham, MA, USA) was used. Full scan MS spectra were acquired at a mass resolution of 60,000 (mass range $350-2000 \mathrm{~m} / \mathrm{z}$ ) in the Orbitrap analyzer. For label-free analyses, tandem mass spectra of the 10 most abundant peaks were acquired in the linear ion trap by peptide fragmentation using collision-induced dissociation (CID). Normalized collision energy (NCE) was set to $35 \%$, and an isolation width of $2 \mathrm{~m} / \mathrm{z}$ was chosen (35-37).

Protein identifications were performed with Proteome Discoverer software. Briefly, Thermo raw-files were imported and searched against UniProt KB/Swiss-Prot database (release 2014_10). For database searches, mass tolerances were set to $10 \mathrm{ppm}$ and $0.8 \mathrm{Da}$ for precursor and fragment ions, respectively. Taxonomy was restricted to human, and one enzymatic miscleavage was allowed. For label-free analyses, modifications of cysteine (carbamidomethyl, static) and methionine (oxidation, variable) were considered. Confidence of peptide identification was estimated using the percolator function, implemented in Proteome Discoverer. Instead of determining the peptide confidence based on a single metric-like Mascot ion score, we decided to use percolator as it discriminates correct from incorrect peptide spectrum matches based on multiple orthogonal score criteria leading to accurate and sensitive peptide identifications. Peptide identifications with false discovery rates $\mathrm{N} 1 \%$ (q-value $\mathrm{N} 0.01$ ) were discarded (35-37). 
Western blot analysis. The gastric cancer tissues, corresponding non-tumor normal tissues and AGS cells were lysed in RIPA buffer (CWBio), and the total protein concentration was determined using Pierce ${ }^{\circledR}$ BCA Protein Assay kit (Thermo Scientific, Inc., Rockford,IL,USA). Extracts containing $50 \mu \mathrm{g}$ of proteins were separated on $10 \%$ SDS-PAGE gels, and electroblotted onto nitrocellulose membranes (HyClone Laboratories, Inc., Logan, UT, USA). The membranes were incubated using Tris-buffered saline/Tween-20 (25 mM Tris- $\mathrm{HCl}, 150 \mathrm{mM}$ $\mathrm{NaCl}, \mathrm{pH} 7.5$ and $0.05 \%$ Tween-20) containing 5\% non-fat milk followed by overnight incubation at $4{ }^{\circ} \mathrm{C}$ with primary antibodies [rabbit anti-CD90 antibody, 1:200; rabbit anti-SPARC antibody, 1:100; rabbit anti-COL1A2 antibody, 1:300 (Wuhan Boster Biological Engineering Co., Ltd., Wuhan, China)]. Following three washes, the membranes were incubated with horseradish peroxidase-conjugated secondary antibodies (Santa Cruz Biotechnology, Inc.), and the specific signals were visualized using an ECL detection system. Anti-GAPDH antibody 1:3,000 (Santa Cruz Biotechnology, Inc.) was used as a loading control.

Statistical analysis. Differences in non-parametric variables were analyzed by the Mann-Whitney U test. Differences in the quantitative variables between groups were analyzed by the Student's t-test using SPSS 11.0 program (SPSS, Chicago, IL, USA). $\mathrm{P}<0.05$ was considered to indicate a statistically significant difference.

\section{Results}

CD90 is highly expressed in the gastric cancer tissues. To detect the mRNA expression levels of the CD90 molecule in gastric cancer and the adjacent non-cancerous tissues, 12 samples of each were selected to perform qPCR of the CD90 gene. The data were analyzed using the $2^{-\Delta \Delta C T}$ method, and the fold-change in expression of these genes relative to the internal control gene, GAPDH, was analyzed. The expression of the CD90 gene was higher in the gastric cancer samples compared with the adjacent non-cancerous tissues and the normalized CD90 gene expression in gastric cancer was upregulated by 3.46 -fold ( $\mathrm{P}=0.0018$ ) (Fig. 1A).

To determine whether the CD90 gene was expressed at a higher level in gastric cancer compared with the adjacent non-cancerous tissues, the protein expression levels of CD90 were further examined by western blot analysis in 4 pair of samples (Fig. 1B). In comparison with the adjacent non-cancerous tissues, the expression level was identified to be higher in the gastric cancer tissues, which corresponded with the qPCR results.

To confirm the pattern of CD90 in gastric cancer, immunohistochemistry (IHC) was carried out with antibodies against CD90 protein in gastric cancer and the adjacent non-cancerous tissues. CD90 was identified as differentially expressed between gastric cancer tissues vs. the adjacent non-cancerous tissues. IHC showed a similar pattern in protein expression with the western blot results. A total of $61.5 \%(16 / 26)$ of the gastric cancer tissues had a high score of CD90 in contrast to $26.9 \%(7 / 26)$ of the adjacent non-cancerous tissues. The distribution of low score was $23.1 \%(6 / 26)$ and $42.3 \%(11 / 26)$ in the gastric cancer and the adjacent non-cancerous tissues,
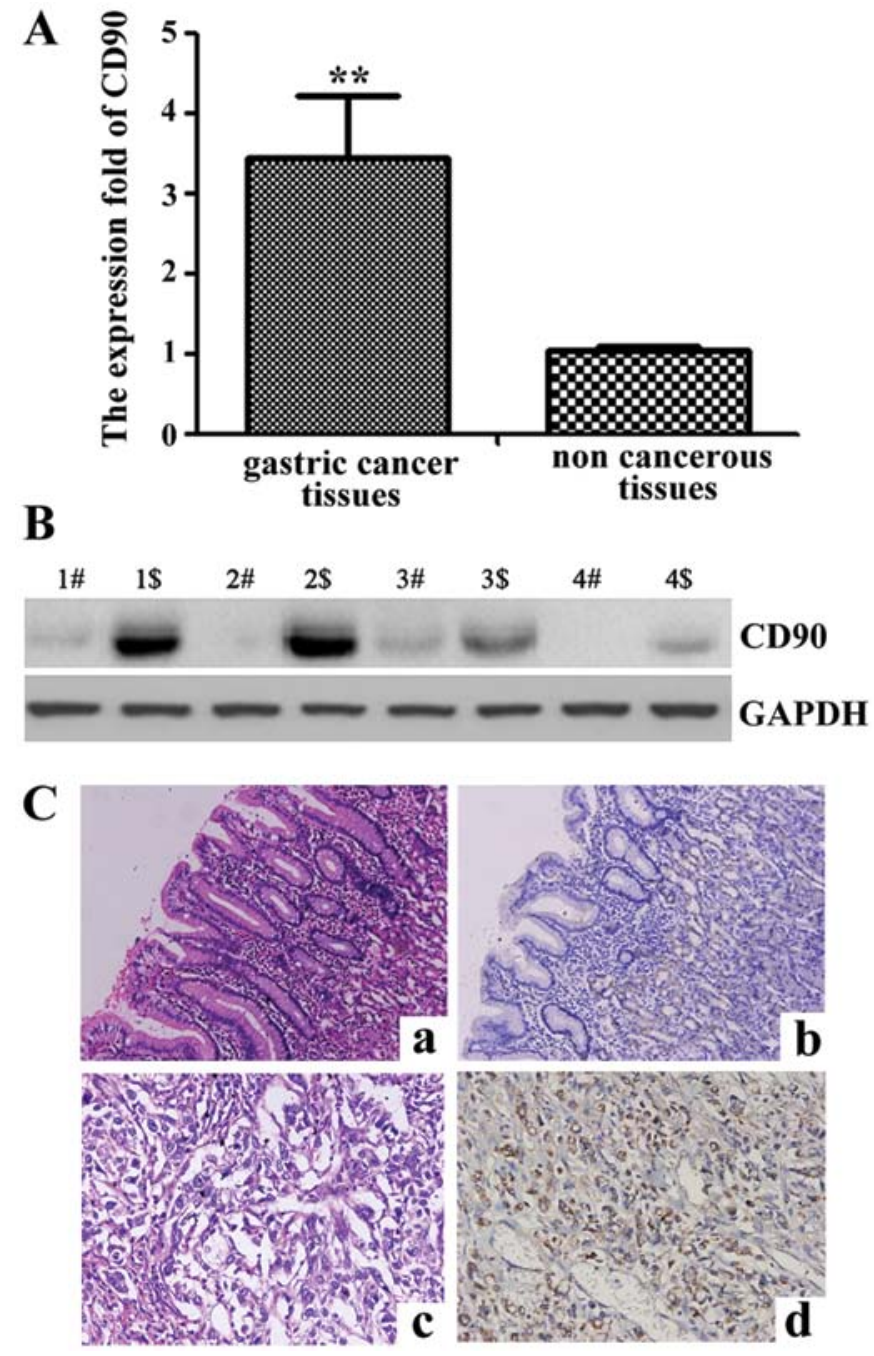

Figure 1. Detection of expression levels of CD90 in the gastric cancer and the adjacent non-cancerous tissues by qPCR, western blot analysis and IHC. (A) qPCR was performed to validate the expression of CD90 in the gastric cancer and adjacent non-cancerous tissues. GAPDH was used as an internal control and for normalization of the data. The mRNA expression level of CD90 was significantly upregulated in the gastric cancer tissues $(\mathrm{n}=12)$ compared with the adjacent non cancerous tissues $(\mathrm{n}=12) .{ }^{* *} \mathrm{P}<0.01$ (B) Four pairs of tissues which were used in the detection of mRNA expression levels by qPCR were selected to detect the expression levels of CD90 protein by western blot analysis. $1 \$-4 \$$, gastric cancer tissues; $1 \#-4 \#$, adjacent non-cancerous tissues. Data are representative of three independent experiments. (C) Immunohistochemical analysis of the expression of CD90 protein in the gastric cancer and adjacent non-cancerous tissues. Antibody of CD90 protein was used. Brown grains denote a positive signal. H\&E staining of gastric epithelial tissue (a), CD90 staining of gastric epithelial tissue (b), $\mathrm{H} \& \mathrm{E}$ staining of gastric cancer tissue (c) and CD90 staining of gastric cancer tissue (d). Original magnification, $\mathrm{x} 200$.

respectively $(\mathrm{P}=0.02)$ (Fig. $1 \mathrm{C}$ and Table II). The results corresponded with the $\mathrm{qPCR}$ results.

CD90 inhibits the apoptosis of gastric cancer cells in vitro. We found that CD90 was highly expressed in gastric cancer tissues by qPCR, western blot analysis and IHC technologies. To elucidate the function of CD90 in the apoptosis of gastric cancer cells, AGS cells were transfected with the plasmid pEGFP-N1/CD90 or the control vector to generate CD90 stable-expressing AGS/CD90 and control AGS/vector cell lines. After assessing CD90 protein by qPCR and 
Table II. Difference in CD90 expression between gastric cancer and the adjacent non-cancerous tissues by immunohistochemistry (IHC).

\begin{tabular}{|c|c|c|c|c|c|c|}
\hline \multirow[b]{2}{*}{ Tissue type } & \multirow[b]{2}{*}{$\mathrm{n}$} & \multicolumn{3}{|c|}{ Score } & \multirow[b]{2}{*}{$\chi^{2}$} & \multirow[b]{2}{*}{ P-value } \\
\hline & & $\begin{array}{c}\text { Low }(0-2) \\
\mathrm{n}(\%)\end{array}$ & $\begin{array}{c}\text { Moderate (3-4) } \\
\mathrm{n}(\%)\end{array}$ & $\begin{array}{l}\text { High (5-6) } \\
\text { n (\%) }\end{array}$ & & \\
\hline Gastric cancer tissues & 26 & $6(23.1)$ & $4(15.4)$ & $16(61.5)$ & 6.33 & 0.04 \\
\hline Non-cancerous tissues & 26 & $11(42.3)$ & $8(30.8)$ & 7 (26.9) & & \\
\hline
\end{tabular}

A

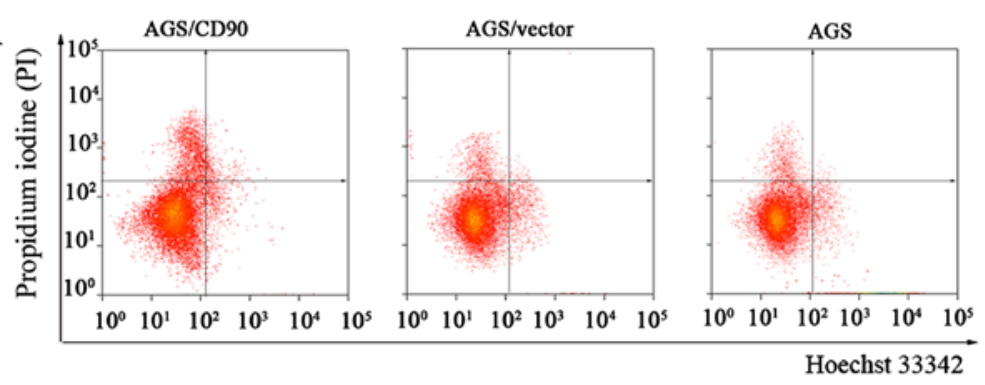

$\mathrm{B}$

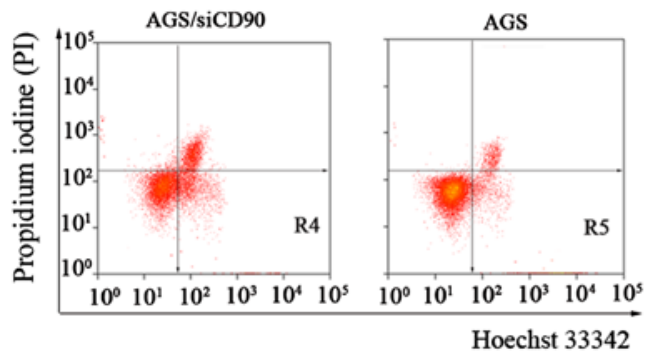

$\mathrm{C}$

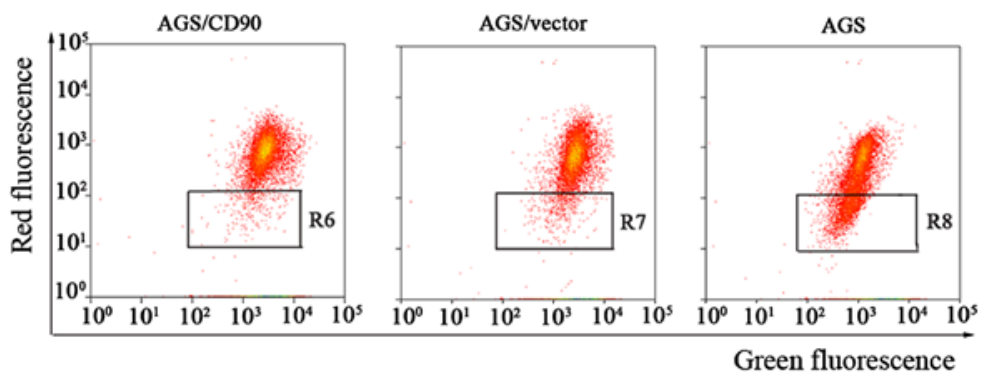

$\mathrm{D}$

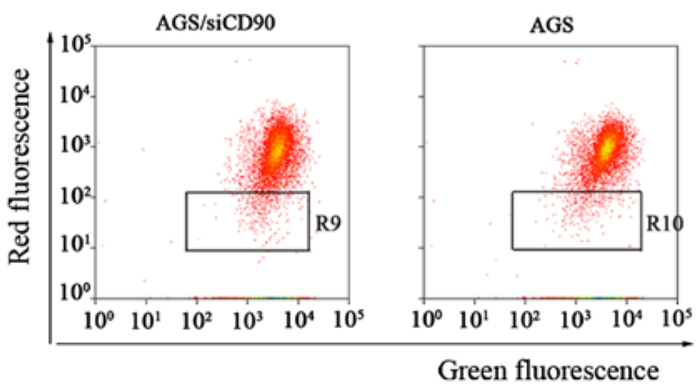

Figure 2. Detection of cell apoptosis and mitochondrial membrane potential $(\Delta \Psi \mathrm{m})$ in gastric cancer cell line, AGS, by flow cytometry. (A) Cell apoptosis of the AGS/CD90, AGS /vector and AGS cells was assessed by flow cytometry. (B) Cell apoptosis of AGS/siCD90 and AGS cells was assessed by flow cytometry. (C) Mitochondrial membrane potential $(\Delta \Psi \mathrm{m})$ of the AGS/CD90, AGS /vector and AGS cells was assessed by flow cytometry. (D) Mitochondrial membrane potential $(\Delta \Psi \mathrm{m})$ of the AGS/siCD90 and AGS cells was assessed by flow cytometry. Data are representative of three independent experiments.

western blot technologies, we performed a Hoechst 33342/PI double-staining experiment to test the rate of apoptosis in the AGS, AGS/vector and AGS/CD90 cells. A considerable decrease in apoptotic cells was observed in the
AGS/CD90 cells ( $5.37 \pm 0.34 \%)$, AGS/vector cells $(10.4 \pm 0.96 \%)$ and AGS cells (11.21 $\pm 0.99 \%)$ (Fig. 2A). At the same time, we detected the effect of the knockdown of the expression level of CD90 (siRNA) and found that the rate of apoptosis was 

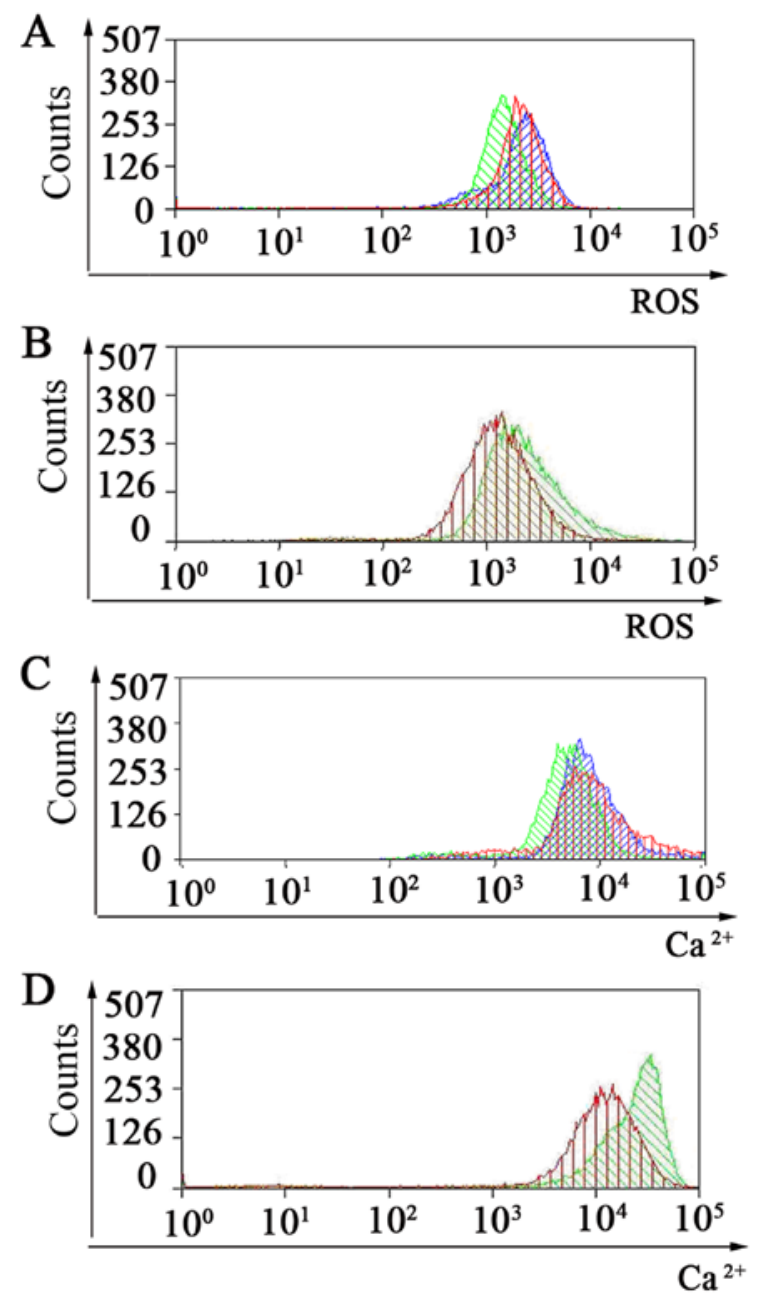

Figure 3. Detection of intracellular reactive oxygen species (ROS) and calcium ion $\left(\mathrm{Ca}^{2+}\right)$ concentrations in gastric cancer cell line, AGS, by flow cytometry. (A) ROS of AGS/CD90, AGS /vector and AGS cells was assessed by flow cytometry. Green indicates AGS/CD90 cells, red indicates AGS cells, blue means AGS /vector cells. (B) ROS of AGS/siCD90 and AGS cells were assessed by flow cytometry. Green indicates AGS/siCD90 cells, red indicates AGS cells. (C) $\mathrm{Ca}^{2+}$ concentrations of AGS/CD90, AGS/vector and AGS cells were assessed by flow cytometry. Green indicates AGS/CD90 cells, red indicates AGS cells, blue indicates AGS /vector cells. (D) $\mathrm{Ca}^{2+}$ concentrations of AGS/siCD90 and AGS cells were assessed by flow cytometry. Green indicates AGS/siCD90 cells, red indicates AGS cells. Data are representative of three independent experiments.

increased in the AGS/siCD90 cells compared with the AGS cells; $15.94 \pm 0.84$ and $10.02 \pm 1.01 \%$ for the AGS/siCD90 and AGS cells, respectively (Fig. 2B).

CD90 affects mitochondrial membrane potential ( $\Delta \Psi \mathrm{m}), \mathrm{ROS}$ and calcium ion $\left(\mathrm{Ca}^{2+}\right)$ concentrations in the gastric cancer cells in vitro. Cell apoptosis is closely related with a reduction in mitochondrial membrane potential $(\Delta \Psi \mathrm{m})$ and an increase in intracellular reactive oxygen species (ROS) and calcium ion $\left(\mathrm{Ca}^{2+}\right)$ concentrations. Thus, we tested the effect of CD90 on these three parameters. Our results showed that overexpression of CD90 in AGS gastric cancer cells, led to an increase in $\Delta \Psi \mathrm{m}$ and inhibited cell apoptosis (Fig. 2C). Moreover, siCD90 reduced the $\Delta \Psi \mathrm{m}$ and induced apoptosis (Fig. 2D). ROS results showed that overexpression of CD90 in the AGS gastric cancer cells, led to a decrease in intracellular reactive oxygen species
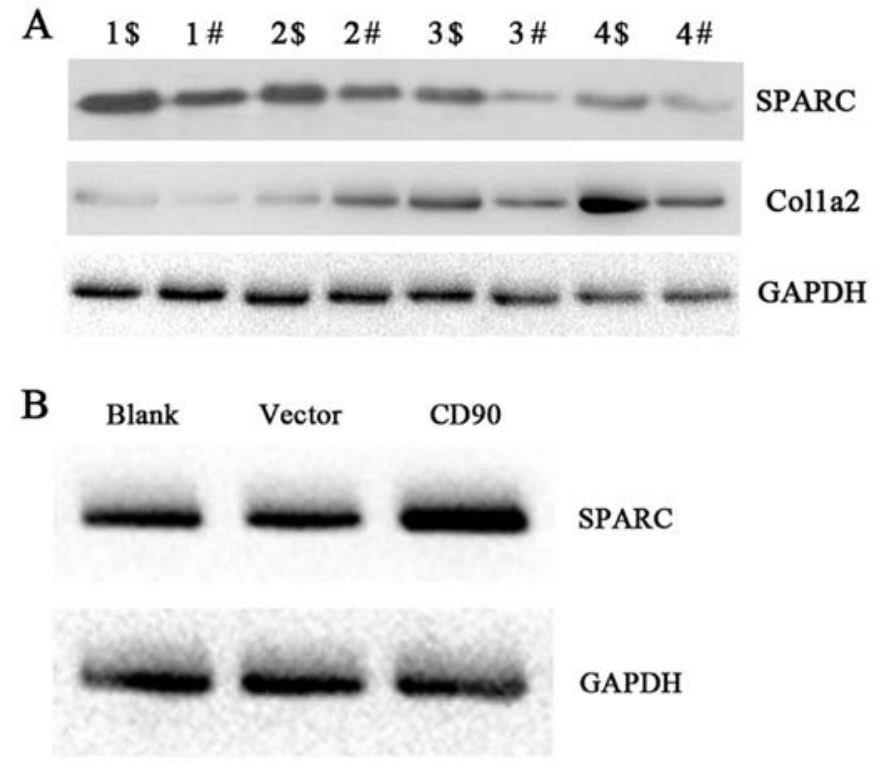

Figure 4. Expression levels of SPARC and Colla2 protein in gastric cancer tissues and a gastric cancer cell line. (A) Four pairs of tissues which were used in the detection of CD90 mRNA expression levels by qPCR were selected to assess the expression levels of SPARC and Col1a2 protein by western blot analysis. 1\$-4\$, gastric cancer tissues; $1 \#-4 \#$, adjacent non-cancerous tissues. Data are representative of three independent experiments. (B) Expression levels of SPARC protein in the AGS gastric cancer cell line. CD90, AGS cells transfected with pEGFP-N1-cd90. Vector, AGS cells transfected with pEGFP-N1. Blank, AGS cells not transfected with the plasmid. Data are representative of three independent experiments.

(ROS) (Fig. 3A). Meanwhile, siCD90 increased the intracellular ROS and induced apoptosis (Fig. 3B). $\mathrm{Ca}^{2+}$ experimental results showed that overexpression of CD90 in the AGS gastric cancer cells led to a decrease in calcium ion $\left(\mathrm{Ca}^{2+}\right)$ concentrations and suppressed the apoptosis of gastric cancer cells (Fig. 3C). Moreover, siCD90 increased calcium ion $\left(\mathrm{Ca}^{2+}\right)$ concentrations and induced apoptosis (Fig. 3D).

CD90 is correlated with dysregulation of SPARC and COL1A2 in gastric cancer tissues. To discover the molecules affected by CD90, we analyzed the genes co-expressed with CD90 by bioinformatic analysis. We found that SPARC, COL1A2 and BGN were the most relevant molecules with CD90. To test the results of the bioinformatic analysis, we tested the expression levels of SPARC and COL1A2 by western blot technology in the gastric cancer and the adjacent non-cancerous tissues. SPARC and COL1A2 were upregulated in the gastric cancer tissues when compared with the levels in the adjacent non-cancerous tissues. Based on the findings that CD90 is highly expressed in gastric cancer, we inferred that CD90 is correlated with the dysregulation of SPARC and COL1A2 in gastric cancer tissues (Fig. 4A).

Identification of differential proteins affected by CD90 through LC-MS/MS analyses. We found that CD90 was highly expressed in gastric cancer tissues and inhibited the apoptosis of gastric cancer cells in vitro. Furthermore, our results suggested that CD90 was correlated with dysregulation of SPARC and COL1A2 in gastric cancer tissues. To confirm that CD90 inhibits cell apoptosis by modulating the 
Table III. The differential proteins in AGS/CD90 and AGS/vector cells affected by CD90 through LC-MS/MS analyses.

\begin{tabular}{|c|c|c|c|c|c|c|c|c|}
\hline \multirow[b]{2}{*}{ No. } & \multicolumn{4}{|c|}{ AGS/CD90 } & \multicolumn{4}{|c|}{ AGS/vector } \\
\hline & Protein & $\begin{array}{l}\text { Unique } \\
\text { peptides }\end{array}$ & $\begin{array}{c}\text { Coverage } \\
(\%)\end{array}$ & $\begin{array}{c}\text { Protein } \\
\text { score }\end{array}$ & Protein & $\begin{array}{l}\text { Unique } \\
\text { peptides }\end{array}$ & $\begin{array}{c}\text { Coverage } \\
(\%)\end{array}$ & $\begin{array}{c}\text { Protein } \\
\text { score }\end{array}$ \\
\hline 1 & FLNB & 48 & 25.05 & 262.99 & EEF1B2 & 44 & 23.05 & 231.28 \\
\hline 2 & EEF1D & 33 & 21.63 & 171.87 & KPNA6 & 42 & 11.23 & 224.82 \\
\hline 3 & HSPA8 & 33 & 19.64 & 132.1 & DECR1 & 30 & 24.68 & 150.08 \\
\hline 4 & CCAR1 & 32 & 9.13 & 114.32 & MGEA5 & 29 & 23.47 & 156.81 \\
\hline 5 & LEPRE1 & 31 & 24.33 & 179.56 & RBM25 & 28 & 43.83 & 119.16 \\
\hline 6 & OGFOD1 & 31 & 25.90 & 157.76 & ACAD9 & 26 & 16.73 & 137.39 \\
\hline 7 & RGPD1 & 30 & 46.99 & 149.72 & OTUB2 & 26 & 16.30 & 72.34 \\
\hline 8 & ACSL3 & 27 & 20.13 & 144.92 & LETM1 & 25 & 44.80 & 153.84 \\
\hline 9 & CTTN & 26 & 45.72 & 184.4 & MRPL43 & 25 & 18.73 & 110.75 \\
\hline 10 & MDH1 & 26 & 14.31 & 158.29 & BCAS2 & 24 & 28.79 & 121.47 \\
\hline 11 & DEK & 24 & 48.59 & 151.03 & PFKFB3 & 23 & 47.65 & 168.64 \\
\hline 12 & NDRG1 & 23 & 17.38 & 132.3 & IARS & 23 & 32.13 & 96.54 \\
\hline 13 & RPL26 & 21 & 26.11 & 104.45 & SART3 & 21 & 37.00 & 175.58 \\
\hline 14 & ACTBL2 & 20 & 36.47 & 158.23 & GATAD2B & 21 & 51.35 & 167.67 \\
\hline 15 & MAGED2 & 20 & 46.78 & 124.36 & NADK2 & 21 & 12.18 & 102.87 \\
\hline 16 & PABPN1 & 20 & 29.51 & 105.72 & TEX9 & 20 & 39.47 & 157.66 \\
\hline 17 & SERPIND1 & 20 & 17.95 & 101.27 & CACNA1S & 20 & 25.64 & 135.74 \\
\hline 18 & BZW1 & 19 & 39.36 & 216.67 & U2SURP & 20 & 35.64 & 107.69 \\
\hline 19 & SIL1 & 19 & 48.45 & 135.76 & SMARCC2 & 20 & 9.62 & 103.38 \\
\hline 20 & TMEM109 & 19 & 35.49 & 117.77 & DCTN2 & 20 & 45.92 & 87.19 \\
\hline 21 & EIF4G2 & 19 & 32.71 & 95.42 & H3F3B & 19 & 39.07 & 107.83 \\
\hline 22 & TRIM25 & 19 & 19.61 & 69.7 & VPS26A & 19 & 14.30 & 88.43 \\
\hline 23 & DSG2 & 18 & 24.66 & 115.12 & ZC3HAV1 & 18 & 38.67 & 246.08 \\
\hline 24 & PDCD4 & 18 & 43.28 & 93.35 & ALDH9A1 & 18 & 57.58 & 143.72 \\
\hline 25 & SNX1 & 18 & 32.02 & 87.33 & UGP2 & 18 & 16.61 & 98.50 \\
\hline 26 & LEPRE1 & 17 & 33.07 & 153.2 & GRPEL1 & 17 & 45.85 & 382.17 \\
\hline 27 & ITGA5 & 17 & 42.52 & 118.48 & ZBTB17 & 17 & 31.98 & 185.06 \\
\hline 28 & SPARC & 17 & 35.95 & 92.55 & CTSA & 17 & 42.52 & 95.30 \\
\hline 29 & AP1B1 & 17 & 7.83 & 91.62 & TNKS1BP1 & 17 & 34.19 & 86.02 \\
\hline 30 & CDC73 & 17 & 22.96 & 84.67 & FASN & 17 & 13.29 & 54.73 \\
\hline
\end{tabular}

Top 30 proteins, ranked by unique peptides.

expression of SPARC and to screen other molecules affected by CD90, we identified the different molecules affected by CD90 through LC-MS/MS analyses. The results showed that there were 132 proteins which were only present in the AGS/CD90 cells and there were 155 proteins which were only present in the AGS/vector cells. According to the frequency of unique peptides, the FLNB protein was the highest in the AGS/CD90 cells, and the following included EEF1D, HSPA8 and CCAR1 (Table III). The SPARC protein was in the list of the top 30 differential proteins in the AGS/CD90 cells when compared with the AGS/vector cells (Table III). EEF1B2 protein was the highest in the AGS/vector cells (Table III).

CD90 affects the expression of SPARC in vitro. To confirm whether CD90 functions by modulating the expression level of SPARC in vitro, we tested the expression of
SPARC protein using CD90 stable-expressing AGS/CD90 and control AGS/vector cell lines by western blot analysis. The results showed that SPARC was upregulated in the AGS cells which overexpressed CD90 (Fig. 4B). Our results suggest that CD90 overexpression affects the expression of SPARC in vivo.

\section{Discussion}

Gastric cancer (GC) is the second most common cause of cancer-related death according to the World Health Organization, and 800,000 cancer-related deaths are caused by GC each year globally (38). Over $70 \%$ of cases occur in developing countries, particularly in East Asian countries (38). Thus, identifying molecular aberrations in GC may improve our understanding of gastric carcinogenesis 
and help us subdivide patients into biologically and clinically relevant subgroups, as well as to develop novel therapeutic strategies.

In the present study, we found that the expression of the CD90 gene was higher in gastric cancer samples compared with that to the adjacent non-cancerous tissues and the normalized CD90 gene expression in gastric cancer was upregulated by 3.46 -fold $(\mathrm{P}<0.01)$; the same trend was found by western blot experiments. IHC showed a pattern in protein expression similar with the qPCR and western blotting results. A total of $61.5 \%(16 / 26)$ of gastric cancer tissues had a high score of CD90 and $26.9 \%(7 / 26)$ of the adjacent non-cancerous tissues. The distribution of low score was $23.1 \%(6 / 26)$ and $42.3 \%(11 / 26)$ in gastric cancer and the adjacent non-cancerous tissues, respectively. Oikonomou et al found that Thy-1 was detected in the majority of 57 gastrointestinal stromal tumor (GIST) samples (54 out of 57 patients, 95\%) and Thy-1-negative patients had a better prognosis. Our results corresponded with these results (13).

To uncover the potential mechanism of CD90 in GC, we studied the effect of CD90 on the apoptosis of AGS gastric cancer cells. Our results showed that there was a considerable decrease in apoptosis in the AGS cells with CD90 overexpression. Compared with the AGS cells, the rate of apoptotic cells was increased in the AGS cells with CD90 interference (siCD90). Thy-1 is a versatile modulator of signaling affecting cellular adhesion, proliferation, survival and cytokine/growth factor responses (39). Thy-1 is an important regulator of cell-cell and cell-matrix interactions, with important roles in nerve regeneration, metastasis, inflammation and fibrosis (1). Previous findings and our results suggest that CD90 affects cell apoptosis.

Cell apoptosis is closely related with a reduction in mitochondrial membrane potential $(\Delta \Psi \mathrm{m})$ and an increase in intracellular ROS and calcium ion $\left(\mathrm{Ca}^{2+}\right)$ concentrations (20-25). In this study, we found that overexpression of CD90 in AGS gastric cancer cells led to an increase in $\Delta \Psi \mathrm{m}$ and a decrease in intracellular ROS and $\mathrm{Ca}^{2+}$ concentrations. Furthermore, siCD90 provided reverse results. Wang et al (40) found that overexpression of Mfn2 induced HepG2 cell apoptosis, reduced the $\Delta \Psi \mathrm{m}$ and endoplasmic reticulum (ER) calcium ion $\left(\mathrm{Ca}^{2+}\right)$ concentrations, and elevated intracellular ROS and mitochondrial $\mathrm{Ca}^{2+}$ concentrations. The rise in the intracellular calcium concentration $\mathrm{Ca}^{2+}$ caused mitochondrial $\mathrm{Ca}^{2+}$ overload, thereby triggering apoptosis (41).

In the present study, we identified and confirmed that CD90 functions by modulated the expression level of SPARC in vitro through LC-MS/MS analyses and western blot technology. SPARC promoter methylation is an important factor in the tumorigenesis of gastric carcinomas and provides new insights into the potential use of SPARC as a novel biomarker and the potential clinical importance in human gastric cancers (42). Overexpression of the SPARC gene may be a useful independent predictor of outcomes in patients with gastric cancer (43). Yin et al found that downregulation of SPARC inhibited the invasion and growth of human gastric cancer cells (44). Thus, the targeting of SPARC could be an effective therapeutic approach against gastric cancer. Wang et al reported the potential of SPARC as a prognostic marker for gastric cancer (45). In summary, our results suggest that CD90 is upregulated in gastric cancer and inhibits gastric cancer cell apoptosis by modulating the expression level of SPARC protein.

\section{Acknowledgements}

This study was supported by the National Natural Science Foundation of China (81272975, 81172302 and 81402270); the Key Project of Hunan Provincial Natural Science Foundation (12JJ2044); the Project of Hunan Provincial Natural Science Foundation (12JJ3121); the Project of Hunan Provincial Development and Reform Commission; the Planned Science and Technology Project of Hunan Province (2010FJ3088 and 2012FJ2014).

\section{References}

1. Rege TA and Hagood JS: Thy-1 as a regulator of cell-cell and cell-matrix interactions in axon regeneration, apoptosis, adhesion, migration, cancer and fibrosis. FASEB J 20: 1045-1054, 2006.

2. Scognamiglio G, D'Antonio A, Rossi G, Cavazza A, Camerlingo R, Pirozzi G, La Mantia E, Anniciello AM, Morabito A, Cantile M, et al: CD90 expression in atypical meningiomas and meningioma metastasis. Am J Clin Pathol 141: 841-849, 2014.

3. Kitayama J, Emoto S, Yamaguchi H, Ishigami H, Yamashita H, Seto Y, Matsuzaki K and Watanabe T: CD90(+)CD45(-) intraperitoneal mesothelial-like cells inhibit $\mathrm{T}$ cell activation by production of arginase I. Cell Immunol 288: 8-14, 2014.

4. Ohmura-Kakutani H, Akiyama K, Maishi N, Ohga N, Hida Y, Kawamoto T, Iida J, Shindoh M, Tsuchiya K, Shinohara N, et al: Identification of tumor endothelial cells with high aldehyde dehydrogenase activity and a highly angiogenic phenotype. PLoS One 9: e113910, 2014.

5. Liu JY, Peng HF, Gopinath S, Tian J and Andreadis ST: Derivation of functional smooth muscle cells from multipotent human hair follicle mesenchymal stem cells. Tissue Eng 16: 2553-2564, 2010.

6. Gorantla VS, Schneeberger S, Moore LR, Donnenberg VS, Zimmerlin L, Lee WP and Donnenberg AD: Development and validation of a procedure to isolate viable bone marrow cells from the vertebrae of cadaveric organ donors for composite organ grafting. Cytotherapy 14: 104-113, 2012.

7. Tang KH, Dai YD, Tong M, Chan YP, Kwan PS, Fu L, Qin YR, Tsao SW, Lung HL, Lung ML, et al: A CD90(+) tumor-initiating cell population with an aggressive signature and metastatic capacity in esophageal cancer. Cancer Res 73: 2322-2332, 2013.

8. Sukowati CH, Anfuso B, Torre G, Francalanci P, Crocè LS and Tiribelli C: The expression of CD90/Thy-1 in hepatocellular carcinoma: An in vivo and in vitro study. PLoS One 8: e76830, 2013.

9. Kawamura K, Hiroshima K, Suzuki T, Chai K, Yamaguchi N, Shingyoji M, Yusa T, Tada Y, Takiguchi Y, Tatsumi K, et al: CD90 is a diagnostic marker to differentiate between malignant pleural mesothelioma and lung carcinoma with immunohistochemistry. Am J Clin Pathol 140: 544-549, 2013.

10. Schubert K, Gutknecht D, Köberle M, Anderegg U and Saalbach A: Melanoma cells use Thy-1 (CD90) on endothelial cells for metastasis formation. Am J Pathol 182: 266-276, 2013.

11. Wandel E, Saalbach A, Sittig D, Gebhardt C and Aust G: Thy-1 (CD90) is an interacting partner for CD97 on activated endothelial cells. J Immunol 188: 1442-1450, 2012.

12. Zhu J, Thakolwiboon S, Liu X, Zhang M and Lubman DM: Overexpression of CD90 (Thy-1) in pancreatic adenocarcinoma present in the tumor microenvironment. PLoS One 9: e115507, 2014.

13. Oikonomou D, Hassan K, Kaifi JT, Fiegel HC, Schurr PG Reichelt U, Aridome K, Yekebas EF, Mann O, Kluth D, et al: Thy-1 as a potential novel diagnostic marker for gastrointestinal stromal tumors. J Cancer Res Clin Oncol 133: 951-955, 2007.

14. Jiang J, Zhang Y, Chuai S, Wang Z, Zheng D, Xu F, Zhang Y, Li C, Liang Y and Chen Z: Trastuzumab (Herceptin) targets gastric cancer stem cells characterized by CD90 phenotype. Oncogene 31: 671-682, 2012.

15. Goldar S, Khaniani MS, Derakhshan SM and Baradaran B: Molecular mechanisms of apoptosis and roles in cancer development and treatment. Asian Pac J Cancer Prev 16: 2129-2144, 2015. 
16. Paul I and Jones JM: Apoptosis block as a barrier to effective therapy in non small cell lung cancer. World J Clin Oncol 5: 588-594, 2014.

17. Beesoo R, Neergheen-Bhujun V, Bhagooli R and Bahorun T: Apoptosis inducing lead compounds isolated from marine organisms of potential relevance in cancer treatment. Mutat Res 768: 84-97, 2014.

18. Li X, Li H, Zhang R, Liu J and Liu J: MicroRNA-449a inhibits proliferation and induces apoptosis by directly repressing E2F3 in gastric cancer. Cell Physiol Biochem 35: 2033-2042, 2015.

19. Marczak A, Denel-Bobrowska $M$, Łukawska $M$ and Oszczapowicz I: Formamidinodoxorubicins are more potent than doxorubicin as apoptosis inducers in human breast cancer cells. Anticancer Res 35: 1935-1940, 2015.

20. Hacker K and Medler KF: Mitochondrial calcium buffering contributes to the maintenance of basal calcium levels in mouse taste cells. J Neurophysiol 100: 2177-2191, 2008.

21. Ogura T, Margolskee RF and Kinnamon SC: Taste receptor cell responses to the bitter stimulus denatonium involve $\mathrm{Ca}^{2+}$ influx via store-operated channels. J Neurophysiol 87: 3152-3155, 2002.

22. Akabas M, Dodd J and al-Awqati Q: Identification of electrophysiologically distinct subpopulations of rat taste cells. J Membr Biol 114: 71-78, 1990.

23. Pulkkinen V, Manson ML, Säfholm J, Adner M and Dahlén SE: The bitter taste receptor (TAS2R) agonists denatonium and chloroquine display distinct patterns of relaxation of the guinea pig trachea. Am J Physiol Lung Cell Mol Physiol 303: L956-L966, 2012.

24. Ruiz-Avila L, McLaughlin SK, Wildman D, McKinnon PJ, Robichon A, Spickofsky N and Margolskee RF: Coupling of bitter receptor to phosphodiesterase through transducin in taste receptor cells. Nature 376: 80-85, 1995.

25. Li Y, Su X, Wang X, Leung AW, Xu C, Wang P and Liu Q Cytotoxic effect of protoporphyrin IX to human leukemia U937 cells under ultrasonic irradiation. Cell Physiol Biochem 33: 1186-1196, 2014

26. Livak KJ and Schmittgen TD: Analysis of relative gene expression data using real-time quantitative PCR and the 2(-Delta Delta C(T)) method. Methods 25: 402-408, 2001.

27. Zhou Y, Wang W, Zheng D, Peng S, Xiong W, Ma J, Zeng Z, Wu M,Zhou M, Xiang J, et al: Risk of nasopharyngeal carcinoma associated with polymorphic lactotransferrin haplotypes. Med Oncol 29: 1456-1462, 2012.

28. Xiao S, Zhou Y, Yi W, Luo G, Jiang B, Tian Q, Li Y and Xue M: Fra-1 is downregulated in cervical cancer tissues and promotes cervical cancer cell apoptosis by p53 signaling pathway in vitro. Int J Oncol 46: 1677-1684, 2015.

29. Zheng D, Liao S, Zhu G, Luo G, Xiao S, He J, Pei Z, Li G and Zhou Y: CD38 is a putative functional marker for side population cells in human nasopharyngeal carcinoma cell lines. Mol Carcinog: Jan 28, 2015 (Epub ahead of print).

30. Liao S, Xiao S, Zhu G, Zheng D, He J, Pei Z, Li G and Zhou Y: CD38 is highly expressed and affects the PI3K/Akt signaling pathway in cervical cancer. Oncol Rep 32: 2703-2709, 2014.

31. Zhu W, Li J, Su J, Li J, Li J, Deng B, Shi Q, Zhou Y and Chen X: FOS-like antigen 1 is highly expressed in human psoriasis tissues and promotes the growth of HaCaT cells in vitro. Mol Med Rep 10: 2489-2494, 2014
32. Hara A and Okayasu I: Cyclooxygenase-2 and inducible nitric oxide synthase expression in human astrocytic gliomas: Correlation with angiogenesis and prognostic significance. Acta Neuropathol 108: 43-48, 2004.

33. Spearman C: The proof and measurement of association between two things. Int J Epidemiol 39: 1137-1150, 2010.

34. Huang W, Sherman BT and Lempicki RA: Systematic and integrative analysis of large gene lists using DAVID bioinformatics resources. Nat Protoc 4: 44-57, 2009.

35. Heo S, Spoerk S, Birner-Gruenberger R and Lubec G: Gel-based mass spectrometric analysis of hippocampal transmembrane proteins using high resolution LTQ Orbitrap Velos Pro. Proteomics 14: 2084-2088, 2014.

36. Haddad T and Kümmerer K: Characterization of photo-transformation products of the antibiotic drug Ciprofloxacin with liquid chromatography-tandem mass spectrometry in combination with accurate mass determination using an LTQ-Orbitrap. Chemosphere 115: 40-46, 2014.

37. Zhang JY, Wang F, Zhang H, Lu JQ and Qiao YJ: Rapid identification of polymethoxylated flavonoids in traditional Chinese medicines with a practical strategy of stepwise mass defect filtering coupled to diagnostic product ions analysis based on a hybrid LTQ-Orbitrap mass spectrometer. Phytochem Anal 25: 405-414, 2014.

38. Jemal A, Bray F, Center MM, Ferlay J, Ward E and Forman D: Global cancer statistics. CA Cancer J Clin 61: 69-90, 2011.

39. Rege TA and Hagood JS: Thy-1, a versatile modulator of signaling affecting cellular adhesion, proliferation, survival, and cytokine/growth factor responses. Biochim Biophys Acta 1763: 991-999, 2006.

40. Wang W, Xie Q, Zhou X, Yao J, Zhu X, Huang P, Zhang L, Wei J, Xie H, Zhou L, et al: Mitofusin-2 triggers mitochondria $\mathrm{Ca}^{2+}$ influx from the endoplasmic reticulum to induce apoptosis in hepatocellular carcinoma cells. Cancer Lett 358: 47-58, 2015.

41. Liu KH, Yang ST, Lin YK, Lin JW, Lee YH, Wang JY, Hu CJ, Lin EY, Chen SM, Then CK, et al: Fluoxetine, an antidepressant, suppresses glioblastoma by evoking AMPAR-mediated calcium-dependent apoptosis. Oncotarget 6: 5088-5101, 2015.

42. Chen ZY, Zhang JL, Yao HX, Wang PY, Zhu J, Wang W, Wang X, Wan YL, Chen SW, Chen GW, et al: Aberrant methylation of the SPARC gene promoter and its clinical implication in gastric cancer. Sci Rep 4: 7035, 2014.

43. Sato T, Oshima T, Yamamoto N, Yamada T, Hasegawa S, Yukawa N, Numata K, Kunisaki C, Tanaka K, Shiozawa M, et al: Clinical significance of SPARC gene expression in patients with gastric cancer. J Surg Oncol 108: 364-368, 2013.

44. Yin J, Chen G, Liu Y, Liu S, Wang P, Wan Y, Wang X, Zhu J and Gao H: Downregulation of SPARC expression decreases gastric cancer cellular invasion and survival. J Exp Clin Cancer Res 29: $59,2010$.

45. Wang CS, Lin KH, Chen SL, Chan YF and Hsueh S: Overexpression of SPARC gene in human gastric carcinoma and its clinic-pathologic significance. Br J Cancer 91: 1924-1930, 2004. 